\title{
SUSCEPTIBILITY TEST FOR FUNGI: CLINICAL AND LABORATORIAL CORRELATIONS IN MEDICAL MYCOLOGY
}

Ana ALASTRUEY-IZQUIERDO(1), Marcia S.C. MELHEM(2), Lucas X. BONFIETTI(2) \& Juan L. RODRIGUEZ-TUDELA(3)

\begin{abstract}
SUMMARY
During recent decades, antifungal susceptibility testing has become standardized and nowadays has the same role of the antibacterial susceptibility testing in microbiology laboratories. American and European standards have been developed, as well as equivalent commercial systems which are more appropriate for clinical laboratories. The detection of resistant strains by means of these systems has allowed the study and understanding of the molecular basis and the mechanisms of resistance of fungal species to antifungal agents. In addition, many studies on the correlation of in vitro results with the outcome of patients have been performed, reaching the conclusion that infections caused by resistant strains have worse outcome than those caused by susceptible fungal isolates. These studies have allowed the development of interpretative breakpoints for Candida spp. and Aspergillus spp., the most frequent agents of fungal infections in the world. In summary, antifungal susceptibility tests have become essential tools to guide the treatment of fungal diseases, to know the local and global disease epidemiology, and to identify resistance to antifungals.
\end{abstract}

KEYWORDS: Susceptibility test; Fungi.

\section{INTRODUCTION}

Fungal infections are a major cause of morbidity and mortality despite the latest developments of diagnostic tools and therapeutic options. Early initiation of the correct antifungal therapy has been demonstrated to have a direct impact on the patient's outcome ${ }^{46}$. More severe infections affect mainly immunocompromised patients but other populations are also infected. New chronic lung infections have been described with a huge impact on the patient's quality of life, and a high cost of treatment and care. Besides, some skin fungal infections involving mucosa and subcutaneous tissues cause substantial morbidity ${ }^{39}$. Cryptococcus, Candida, Aspergillus, and Pneumocystis are the main etiologic agents of fungal infections ${ }^{12}$. The burden and mortality associated with these diseases depend on the region and the affected population. Thus, it has been estimated that cryptococcal meningitis affects nearly one million people per year. Despite treatment, mortality rates can reach 55 to $70 \%$ in AIDS patients in Latin America and sub-Saharan Africa, the estimated number of deaths per year being over $620,000^{45}$.

Cryptococcus, Candida, Aspergillus, and Pneumocystis affect mainly immunocompromised individuals, however endemic dimorphic fungi such as Histoplasma, Blastomyces, Coccidioides and Paracoccidioides affect immunocompetent patients as well, and endemic areas include several regions of Latin America ${ }^{24}$.
Nowadays, three main families of antifungals are used in the clinical setting to treat fungal infections: polyenes represented by amphotericin B (and its different formulations); azoles with several derivatives such as itraconazole, fluconazole, voriconazole, posaconazole, isavuconazole; and the echinocandins caspofungin, micafungin and anidulafungin. The availability of new antifungals in recent years has provided clinicians with more options, increasing the use of these compounds not just for treatment when the infection has been diagnosed, but also as prophylactic, empirical or preemptive treatment. The increased use of antifungals has induced a higher selective pressure on fungal strains and resistance has emerged in two main ways: several species have developed secondary resistance and susceptible species have been replaced by resistant ones, changing the epidemiology of fungal infections ${ }^{41}$.

Antifungal susceptibility testing methods are available to detect antifungal resistance and to determine the best treatment for a specific fungus. Clinical microbiology relies on these methods to select the agent of choice for a fungal infection, and to know the local and the global epidemiology of antifungal resistance.

Microdilution methods are the gold standard or reference techniques. Two organizations, the European Committee on Antibiotic Susceptibility Testing (EUCAST) and the Clinical Laboratory Standards Institute (CLSI) have standardized methods to perform antifungal susceptibility testing. Differences between these two methods have been widely 
ALASTRUEY-IZQUIERDO, A.; MELHEM, M.S.C.; BONFIETTI, L.X. \& RODRIGUEZ-TUDELA, J.L. - Susceptibility test for fungi: clinical and laboratorial correlations in medical mycology. Rev. Inst. Med. Trop. Sao Paulo, 57(Suppl 19): 57-64, 2015.

discussed in several reports and will be reviewed in the present manuscript, however their results have demonstrated to be comparable and are used worldwide. Both institutions have developed breakpoints (BPs) of some antifungals to Candida and Aspergillus species that are currently used to classify resistant strains.

Regardless of their advantages, the standardized broth microdilution methods of antifungal susceptibility testing are time-consuming and cumbersome for clinical laboratories. Some commercially available, including manual, semi-automated and automated methods, do not require complex handling and are cost-effective alternative methods to test antifungal agents in vitro against Candida isolates in routine usage, and Cryptococcus isolates and filamentous fungi for research purposes. The characteristics of these methods together with their comparison with the reference procedures and the available agar-based methods will also be reviewed in this manuscript.

\section{REFERENCE METHODS}

\section{Broth microdilution methods}

\section{Clinical Laboratory Standards Institute (CLSI)}

In 1985, the CLSI, formerly known as the National Committee for Clinical Laboratory Standards (NCCLS), formed a subcommittee on Antifungal Susceptibility Testing that published, in 1997, the document M27A "Reference Method for Broth Dilution Antifungal Susceptibility Testing of Yeast; Approved Standard"19. This document defined reference strains with ranges of Minimal Inhibitory Concentrations (MIC) and Break Points (BPs) for some antifungals and their action against yeasts such as Candida and Cryptococcus. Since then, several updates have been published, the current one having been approved in April $2008^{20}$. For filamentous fungi, the first document was published in 2002: M38A: "Reference Method for Broth Dilution Antifungal Susceptibility Testing of Filamentous Fungi; Approved Standard" with a second edition published in 2008, which is the currently accepted one ${ }^{21}$.

\section{European Committee on Antimicrobial Susceptibility Testing (EUCAST)}

The EUCAST is a standing committee jointly organized by the European Society of Clinical Microbiology and Infectious Diseases (ESCMID), the European Centre for Diseases Control (ECDC), and the European National Breakpoint Committees (www.eucast.org). The antifungal susceptibility testing subcommittee of the EUCAST (AFSTEUCAST) was formed in 1997, and in 2008 published a standard of susceptibility testing for yeasts (including Cryptococcus). This standard was updated in $2012^{6}$. Another standard for molds was published in 2008. All of these standards are available online and can be downloaded from the EUCAST website (www.eucast.org). Differences between both standards are mainly found in the inoculum size, incubation time and medium composition (Table 1). Despite these differences, the results obtained by both methods are comparable ${ }^{15,55}$. CLSI and EUCAST include in their standards to test yeast some modifications for Cryptococcus. Thus, in CLSI the recommendation is to read MICs for Cryptococcus after 70 to 74 hours of incubation (in contrast with $24-48 \mathrm{~h}$ for Candida), while EUCAST recommends the incubation of the plates at $30^{\circ} \mathrm{C}$ when the growth control does not reach an optical density of 0.2 at $35^{\circ} \mathrm{C}$. Neither
CLSI nor EUCAST have published standards for endemic dimorphic fungi such as Histoplasma or Paracoccidioides.

Table 1

CLSI vs EUCAST methodologies for antifungal susceptibility testing

\begin{tabular}{lcc}
\hline & CLSI M27-A3 & EUCAST Edef 7.2 \\
\hline Format & Microdilution & Microdilution \\
Well shape (bottom) & round & flat \\
Media & RMPI 1640 & RPMI 1640 \\
Glucose content & $\mathbf{0 . 2 \%}$ & $\mathbf{2 \%}$ \\
Inoculum size & $0.5-2.5 \times 10^{3}$ & $0.5-2.5 \times 10^{5}$ \\
Incubation temperature & $35^{\circ} \mathrm{C}$ & $35^{\circ} \mathrm{C}$ \\
Incubation time & & \\
AMB, FCZ, Candins & $24 \mathrm{~h}$ & $24 \mathrm{~h}$ \\
Azoles & $\mathbf{4 8 h}$ & $\mathbf{2 4 h}$ \\
Cryptococcus & $\mathbf{7 2 h}$ & $\mathbf{4 8 h}$ \\
Reading & Visual & Spectrophotometric
\end{tabular}

Endpoint

AMB

Azoles and Candins
$100 \%$ inhibition

$50 \%$ inhibition
90\% inhibition

$50 \%$ inhibition
Differences between two methods are in bold. $\mathrm{AMB}=$ amphotericin $\mathrm{B} ; \mathrm{FCZ}=$ fluconazole; Candins $=$ anidulafungin, caspofungin, micafungin .

\section{Agar-based methods}

Disk tests are inexpensive and easy to set up, and provide an ideal screening test. The disk diffusion method to test antifungals (CLSI M44 series) has been developed and validated only in the case of azoles and echinocandins for Candida spp. isolates ${ }^{16}$. It recommends the use of Mueller-Hinton agar supplemented with $2 \%$ glucose, providing a suitable growth for most yeasts, and $0.5 \mathrm{mg} / \mathrm{L}$ methylene blue dye medium (enhances the zone edge definition) minimizing the trailing effect. The $\mathrm{pH}$ of the medium needs to be between 7.2 and 7.4 after gelling and the agar should be $4 \mathrm{~cm}$ high. The inoculum is standardized to $0.5 \mathrm{McFarland}$ using a densitometer and plates should be incubated at $35^{\circ} \mathrm{C}$ for $24 \mathrm{~h}$; some strains show insufficient growth and may need $48 \mathrm{~h}$ of incubation. In addition, quality control parameters have been established following the CLSI standard procedures. The results of the susceptibility test according to the zone diameter interpretative criteria for caspofungin, fluconazole and voriconazole for Candida species allows to classify the isolate in one of the following categories: susceptible, resistant, susceptible dose dependent and non-susceptible, corresponding to MIC breakpoints ${ }^{23}$.

The essential agreement between the disk diffusion and the CLSI microdilution method to test the susceptibility of azoles against Candida and Cryptococcus isolates is usually higher than $90 \%$ demonstrating that the disk diffusion is able to identify resistant isolates ${ }^{11,35}$. Regarding echinocandins and Candida species, the disk diffusion test appears to be able to differentiate caspofungin-susceptible between resistant mutant isolates. However, the disk diffusion test for micafungin appears to be less optimal due to a close overlapping of susceptible and resistant 


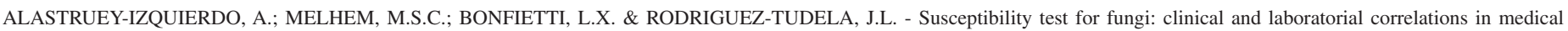
mycology. Rev. Inst. Med. Trop. Sao Paulo, 57(Suppl 19): 57-64, 2015.

populations. In the case of $C$. parapsilosis and C. glabrata, there is a need for individual breakpoints. This behavior has been observed with either EUCAST or CLSI microdilution methods and thus appears to be drugrelated rather than dependent on the choice of the in vitro susceptibility test format. Nevertheless, while susceptibility classification is improved by the application of recently revised breakpoints, further evaluation and refinement are needed ${ }^{8,9}$.

The standard disk diffusion method to test antifungal drugs for non-dermatophyte filamentous fungi isolates (M51-A and supplement M51-S1) provides qualitative results in 8-24 h when caspofungin, triazoles, and amphotericin B are used, faster than the CLSI reference microdilution method ${ }^{17}$. Among Aspergillus species, a lower agreement of results produced by disk diffusion susceptibility tests was reported for A. flavus and amphotericin B or voriconazole. Amphotericin B to test $A$. fumigatus susceptibility also showed a lower agreement when compared to the reference method. Amphotericin B disks usually show the lowest correlation between MICs and inhibition zone diameters for filamentous fungi. The percentage of major errors is usually similar to that obtained with the itraconazole disk, but the percentage of minor errors is higher ${ }^{34,38}$. Although breakpoints for filamentous fungi have not been defined, epidemiological cut-off values can be proposed to identify non-wild-type isolates ${ }^{18}$.

Although qualitative results provided by the disk diffusion method are useful in the clinical laboratory routine, quantitative MIC data is somewhat critical for the management of invasive infections.

\section{Breakpoints}

Even though the main goal of AFST is to select the best treatment for a given isolate, these methods are also very important to detect resistant strains, allowing the establishment of an epidemiology map of antifungals resistance that is an emerging problem in medical mycology. The two main factors are: the development of secondary resistance and the selection of species that are intrinsically resistant. Therefore, AFST has become critical for the choice of the best antifungal agent. Breakpoints have been developed for some fungal species and antifungals, in both CLSI and EUCAST methods. These BPs categorize fungal isolates into (i) susceptible (the drug is an appropriate treatment); (ii) resistant (the drug is not recommended as a treatment), and (iii) intermediate (the drug may be an appropriate treatment, depending on certain conditions; e.g. fluconazole to treat a urinary infection caused by an intermediate strain).

BPs definition is a complex process based on the critical review of several aspects and data. CLSI evaluates MIC distributions, the relationship between MICs and clinical outcome, pharmacokinetics and pharmacodynamics. CLSI proposed a single interpretative BPs for fluconazole, itraconazole ${ }^{19}$, voriconazole ${ }^{52}$, and echinocandins ${ }^{50}$ for all Candida species. Latter, CLSI BPs was revised including a number of clinical studies and cases reporting strains classified as susceptible but associated with treatment failure, and as a consequence, species-specific BPs were proposed, as had been previously established by EUCAST ${ }^{22,49}$.

EUCAST evaluates five aspects to develop BPs: (i) The most common dosage used in each European country; (ii) the definition of the wild type population for each target microorganism at the species level, and the determination of epidemiological cut-offs; (iii) the pharmacokinetics of the drug; (iv) the pharmacodynamics including Monte Carlo simulations; and (v) the correlation of MICs with patients' clinical outcome treated with this drug. Clinical BPs have been established for several antifungals for Candida spp. and Aspergillus spp. These BPs are freely available online at: www.eucast.org/clinical_breakpoints.

As stated before, although some differences have been recognized for several years, currently CLSI and EUCAST breakpoints are in agreement. Tables 2 and 3 represent the established BPs for several antifungal agents for Candida and Aspergillus in both standards.

\section{Resistance}

Antifungal resistance is becoming an emerging problem. On the one hand, there is the intrinsic resistance, and on the other hand the development of secondary resistance, that should be detected because resistant strains are associated with poorer outcomes. To illustrate this problem, intrinsic resistance of $C$. glabrata and $C$. krusei to fluconazole is well known. In these cases, appropriate treatment can be decided on the basis of species identification 7 . This intrinsic resistance has justified the use of echinocandins as primary treatment, instead of fluconazole, in the empirical treatment of candidemia and invasive candidiasis in recently published guidelines ${ }^{25,58}$. In addition, intrinsic resistance to echinocandins has been described in C. parapsilosis, and Cryptococcus neoformans isolates ${ }^{59}$. Although it is less common, during antifungal therapy acquired resistance in Candida spp. infections has also been reported. Most cases involve $C$. glabrata resistance to echinocandin although other species such as $C$. albicans, C. tropicalis and C. krusei, have also proven able of developing secondary resistance ${ }^{31,37}$. Alterations on genes encoding the target enzymes of these drugs (beta 1-3 D-glucan synthase for echinocandins (FKS) and 14 alpha sterol demethylase for azoles (ERG11) or up regulation of multidrug efflux transporters also for azoles (ABC [ATP-binding cassette]/ MFS [major facilitator superfamily]) have been blamed for the Candida spp. resistance to antifungal agent. Point mutations located at two hot spot regions within the FKS genes of Candida spp. have been described and associated with echinocandins resistance ${ }^{53}$.

Secondary resistance to amphotericin B has been described in C. tropicalis, C. parapsilosis, C. lusitaniae, and C. haemulonii ${ }^{33}$. In addition, several emerging pathogens such as A. terreus, Fusarium spp, and Lomentospora prolificans (syn. Scedosporium prolificans) are intrinsically resistant to amphotericin $\mathrm{B}^{2,30}$. The mechanism of resistance to amphotericin $\mathrm{B}$ has been associated with a decrease of ergosterol content in fungal membranes, mainly due to alterations in the ergosterol biosynthesis pathway. It has also been suggested that resistance to amphotericin $\mathrm{B}$ could be related to disruption of the fungal mitochondria ${ }^{44}$.

The azole resistance of Aspergillus isolates has been rigorously investigated in the last years. Alterations in the coding region of the cyp51A gene (positions G54, G138, M220, G448) or an insertion of a 34 to 36 base pair tandem repeat in the promoter region of the gene, together with point mutations (positions L98, Y121 and T289) have been associated with azole resistance. Mechanisms of azole resistance have been described both prior to triazole exposure and acquired during therapy ${ }^{4}$. The use of azoles in agriculture has been described as a cause of the emergence of triazole resistant in Aspergillus fumigatus isolates, particularly in Europe and $\mathrm{Asia}^{56}$. 
ALASTRUEY-IZQUIERDO, A.; MELHEM, M.S.C.; BONFIETTI, L.X. \& RODRIGUEZ-TUDELA, J.L. - Susceptibility test for fungi: clinical and laboratorial correlations in medical mycology. Rev. Inst. Med. Trop. Sao Paulo, 57(Suppl 19): 57-64, 2015.

Table 2

EUCAST and CLSI antifungal breakpoints for Candida

\begin{tabular}{|c|c|c|c|c|c|c|c|c|c|c|c|}
\hline \multirow{3}{*}{$\begin{array}{l}\text { Antifungal } \\
\text { agent }\end{array}$} & \multirow{3}{*}{ Standard } & \multicolumn{10}{|c|}{ MIC breakpoint $(\mathrm{mg} / \mathrm{L})$} \\
\hline & & \multicolumn{2}{|c|}{ C. albicans } & \multicolumn{2}{|c|}{ C. glabrata } & \multicolumn{2}{|c|}{ C. krusei } & \multicolumn{2}{|c|}{ C. parapsilosis } & \multicolumn{2}{|c|}{ C. tropicalis } \\
\hline & & $\mathrm{S} \leq$ & $\mathrm{R}>$ & $\mathrm{S} \leq$ & $\mathrm{R}>$ & $\mathrm{S} \leq$ & $\mathrm{R}>$ & $\mathrm{S} \leq$ & $\mathrm{R}>$ & $\mathrm{S} \leq$ & $\mathrm{R}>$ \\
\hline \multirow[t]{2}{*}{ AMB } & EUCAST & 1 & 1 & 1 & 1 & 1 & 1 & 1 & 1 & 1 & 1 \\
\hline & CLSI & - & - & - & - & - & - & - & - & - & - \\
\hline \multirow[t]{2}{*}{ ANF } & EUCAST & 0.03 & 0.03 & 0.06 & 0.06 & 0.06 & 0.06 & 0.002 & 4 & 0.06 & 0.06 \\
\hline & CLSI & 0.25 & 0.5 & 0.12 & 0.25 & 0.25 & 0.5 & 2 & 4 & 0.25 & 0.5 \\
\hline \multirow[t]{2}{*}{$\mathrm{CPF}$} & EUCAST & & & & & & & - & - & & \\
\hline & CLSI & 0.25 & 0.5 & 0.125 & 0.25 & 0.25 & 0.5 & 2 & 4 & 0.25 & 0.5 \\
\hline \multirow[t]{2}{*}{ FCZ } & EUCAST & 2 & 4 & 0.002 & 32 & - & - & 2 & 4 & 2 & 4 \\
\hline & CLSI & 2 & 4 & $32(\mathrm{SDD})$ & 32 & - & - & 2 & 4 & 2 & 4 \\
\hline \multirow[t]{2}{*}{ ICZ } & EUCAST & 0.06 & 0.06 & IE & $\mathrm{IE}$ & IE & $\mathrm{IE}$ & 0.12 & 0.12 & 0.12 & 0.12 \\
\hline & CLSI & 0.125 & 0.5 & 0.125 & 0.5 & 0.125 & 0.5 & 0.125 & 0.5 & 0.125 & 0.5 \\
\hline \multirow[t]{2}{*}{$\mathrm{MCF}$} & EUCAST & 0.016 & 0.016 & 0.03 & 0.03 & IE & $\mathrm{IE}$ & 0.002 & 2 & IE & IE \\
\hline & CLSI & 0.25 & 0.5 & 0.06 & 0.12 & 0.25 & 0.5 & 2 & 4 & 0.25 & 0.5 \\
\hline \multirow[t]{2}{*}{$\mathrm{PCZ}$} & EUCAST & 0.06 & 0.06 & IE & IE & IE & $\mathrm{IE}$ & 0.06 & 0.06 & 0.06 & 0.06 \\
\hline & CLSI & - & - & - & - & - & - & - & - & - & - \\
\hline \multirow[t]{2}{*}{$\mathrm{VCZ}$} & EUCAST & 0.125 & 0.125 & IE & IE & IE & IE & 0.125 & 0.125 & 0.125 & 0.125 \\
\hline & CLSI & 0.125 & 0.5 & & & 0.5 & 1 & 0.125 & 0.5 & 0.125 & 0.5 \\
\hline
\end{tabular}

SDD = susceptible dose dependant; IE = insufficient evidence.

Table 3

EUCAST antifungal breakpoints for Aspergillus

\begin{tabular}{|c|c|c|c|c|c|c|c|c|c|c|}
\hline \multirow{3}{*}{ Antifungal agent } & \multicolumn{10}{|c|}{ MIC breakpoint (mg/L) } \\
\hline & \multicolumn{2}{|c|}{ A. flavus } & \multicolumn{2}{|c|}{ A. fumigatus } & \multicolumn{2}{|c|}{ A. nidulans } & \multicolumn{2}{|c|}{ A. niger } & \multicolumn{2}{|c|}{ A. terreus } \\
\hline & $\mathrm{S} \leq$ & $\mathrm{R}>$ & $\mathrm{S} \leq$ & $\mathrm{R}>$ & $\mathrm{S} \leq$ & $\mathrm{R}>$ & $\mathrm{S} \leq$ & $\mathrm{R}>$ & $\mathrm{S} \leq$ & $\mathrm{R}>$ \\
\hline Amphotericin B & - & - & 1 & 2 & - & - & 1 & 2 & - & - \\
\hline Itraconazole & 1 & 2 & 1 & 2 & 1 & 2 & - & - & 1 & 2 \\
\hline Posaconazole & - & - & 0.125 & 0.256 & - & - & - & - & 0.125 & 0.256 \\
\hline Voriconazole & - & - & 1 & 2 & - & - & - & - & - & - \\
\hline
\end{tabular}

Other filamentous fungi are intrinsically resistant to some antifungals. The order mucorales comprises several pathogenic species that are resistant to voriconazole ${ }^{1}$. Species of the genera Fusarium and Scedosporium also show elevated MICs of several antifungals ${ }^{3,26}$. In addition, multi-resistant species are also present as human pathogens. Lomentospora prolificans (syn. Scedosporium prolificans) is resistant to all azoles, echinocandins and amphotericin B, and has been associated with poorer outcomes ${ }^{54}$.

\section{Commercial methods}

Clinical laboratories can determine susceptibility to antifungals through a series of commercially available systems, including the Sensititre YeastOne ${ }^{\circledR}$ panel (TREK Diagnostic Systems, Cleveland, USA) and the Vitek 2 system, both based on microdilution methods, or agar-based assays, e.g. test strips (E-Test ${ }^{\circ}$, bioMérieux; MIC $®$, Oxoid) and discs impregnated with a single antifungal agent.

In order to choose a commercial method, first of all, the laboratory should be aware of the commercial techniques results considering the susceptibility of each drug to a particular fungus, comparing the CLSI and the EUCAST reference procedures. In general, the correlation is based on the essential agreement (EA), defined as the discrepancies between MIC results of no more than \pm 2 twofold dilutions, and the categorical 


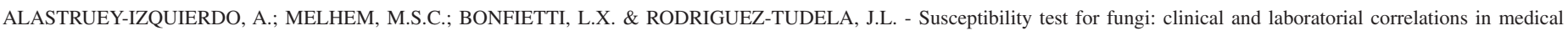
mycology. Rev. Inst. Med. Trop. Sao Paulo, 57(Suppl 19): 57-64, 2015.

agreements (CA). The latter depends on the existence of interpretative break points $\mathrm{s}^{27,48}$. Of note, the laboratory should perform tests strictly as instructed in the commercial guidelines to get reliable results. Additionally, quality control strains, such as C. krusei ATCC 6258 and $C$. parapsilosis ATCC 22019 must be included in each commercial system batch, and be ascertain that all MIC values are within the expected ranges.

\section{Commercial broth microdilution methods}

Sensititre YeastOne ${ }^{\circledR}$ is a well-described colorimetric microdilution panel that contains dried serial twofold dilutions of up to ten antimycotics in individual wells. YeastOne ${ }^{\circledR}$ provides customizable dual-isolate five antifungal format for Candida spp. (clinical use), and single-isolate, nine antifungal, research-use-only format including anidulafungin and micafungin to be tested for yeast and filamentous fungi (not for use in diagnostic procedures). The susceptibility of isolates to antimycotics is assessed on the basis of growth or inhibition of the isolate in the culture media containing antimycotic agents. The system incorporates Alamar Blue ${ }^{\circledR}$, a colorimetric indicator of an oxidation-reduction reaction (fungal growth changes media color from blue to pink). Endpoint determination was based on visual reading or software-facilitated visual reading (Vizion ${ }^{\circledR}$ system) after 24-25 h (Candida spp.) or 48-72h (Cryptococcus spp.) of incubation at $35{ }^{\circ} \mathrm{C}$. The panel has the advantage of being ready to use, easy to perform, quick and timely results, and individual packaging allows the test of one plate at a time. The Sensititre Yeast One ${ }^{\circledR}$ method showed good results in terms of reproducibility and agreement with reference methods considering fluconazole and Candida spp. (EA $\geq 95 \%)$ although a lower agreement (EA 79-92\%) was found to $C$. neoformans isolates. YeastOne ${ }^{\circledR}$ panel was reported to yield higher MICs, in comparison with the CLSI method, for all drugs except for caspofungin and flucytosine ${ }^{5}$. With respect to filamentous fungi, a strong correlation with the M38-A2 (CLSI) method was found for itraconazole and voriconazole. The method showed a strong correlation with CLSI to detect resistant isolates and may help to monitor the emergence of isolates with decreased susceptibility to antifungal agents.

Another commercially available system, called SensiQuattro Candida EU® (bestbiondx, Germany), correlates well with the antifungal clinical break points established by EUCAST. This 32-well colorimetric microdilution panel includes four doubling serial concentrations of amphotericin B, fluconazole, voriconazole, posaconazole, caspofungin, anidulafungin, micafungin, and flucytosine. The resulting colors are interpreted as follows: a yellow/orange color indicates yeast growth; a red color indicates yeast growth inhibition. When compared to the EUCAST reference, the broth microdilution method showed a good correlation for amphotericin B and azoles, but poor for echinocandins ${ }^{40}$.

Vitek $2 ®$ yeast susceptibility test (bioMérieux, Inc.) is an automated method of yeast species identification and antifungal susceptibility testing through the analysis of yeast growth. The system provides 64-well cards containing aliquots of amphotericin B, fluconazole, flucytosine, and voriconazole in a miniaturized version of the broth dilution method. The system integrates a software program which validates and interprets susceptibility test results according to CLSI clinical breakpoints based on the drug MIC values. The high level of standardization achieved by this automated system was demonstrated in several studies ${ }^{5,13,27}$. The number of hours to deliver an MIC result was reported to vary from $9.1 \mathrm{~h}$ to $15 \mathrm{~h}$ for Candida species (minimum $7.5 \mathrm{~h}$ to maximum $18 \mathrm{~h}$ ) and 12.1 h for C. neoformans ${ }^{5,13,27}$. In general, the MICs obtained by the Vitek $2 \circledR$ system are slightly higher than those generated by the CLSI methodology for both Candida and Cryptococcus. However, Vitek $2 \circledR$ results are reproducible, accurate, present a strong correlation with those obtained with the CLSI and the AFST-EUCAST reference methods for fluconazole, amphotericin B, flucytosine, and voriconazole. The correlation with the reference methods was also very good when resistance to antifungals was studied ${ }^{43,47,51}$

\section{Commercial agar-based methods}

Commercially prepared strips are available from bioMérieux (Etest $\left.{ }^{\circledR}\right)$ and Liofilchem Diagnostici (MIC Test Strip®). The method consists of a predefined gradient of antifungal drug concentrations on a plastic strip that is used to determine the MIC. When the strip is applied on an inoculated agar surface, the antifungal agent is immediately transferred to the agar matrix and after an incubation time, an inhibition ellipse centered along the strip is formed. The recommended agar is RPMI 1640 supplemented with $2 \%$ glucose, prepared with MOPS in a $1.5 \%$ agar base. The Etest ${ }^{\circledR}$ provides strips with fluconazole, itraconazole, amphotericin $\mathrm{B}$, flucytosine, voriconazole, posaconazole, and caspofungin. The MIC Test Strips ${ }^{\circledR}$ contains the same antifungal drugs plus anidulafungin, micafungin and ketoconazole. The incubation time range from $24-48 \mathrm{~h}$ for Candida species, from 48-72 h for C. neoformans, and for filamentous fungi it lasts $16 \mathrm{~h}$ or longer depending on the fungus' genus. The MIC is read directly from the scale at the point where the edge of the ellipse intersects the strip. However, it is important to consider that, as for any test evaluating the antimicrobial susceptibility, the medium formulation and, in this case, the depth of the agar can strongly influence MIC results. Therefore, the manufacturer's recommendations should be strictly followed to obtain MICs using strips ${ }^{16,17,21}$.

Results obtained by the E-test method shows a $>71 \%$ correlation with those obtained by the AFST-EUCAST method. In both methods, the CLSI and EUCAST AFST, the agar-based E-test has been proposed as a more sensitive technology to discriminate strains of Candida species with $f k s$ mutations from wild-type (WT) strains by virtue of much higher MIC results observed in mutant strain. Considering Cryptococcus, the overall agreement level using the E-test MICs and the EUCAST AFSTMICs seems to be higher for voriconazole, fluconazole, itraconazole and flucytosine, than for amphotericin B, which has the lowest level of agreement. Regarding filamentous fungi, the agreement is higher for itraconazole than for amphotericin B, and the E-test method showed a good correlation with the CLSI M38-AFST one to detect Aspergillus resistance. Systematic comparisons between MIC results from reference laboratories and routine results obtained using commercially available methods could be more representative than the current practice to perform quality control with a specific set of reagents using a limited number of isolates ${ }^{14}$.

\section{CONCLUSIONS}

The role of microdilution methods seems to be restricted to reference laboratories because they are laborious. In addition, the microbroth format is not commonly used in clinical laboratories. Several automated or semiautomated commercial methods based on agar diffusion or the use of colorimetric indicators in Etest, Sensititre YeastOne, Fungitest or Vitek have been designed for routine daily practice. Disk and strip diffusion 
ALASTRUEY-IZQUIERDO, A.; MELHEM, M.S.C.; BONFIETTI, L.X. \& RODRIGUEZ-TUDELA, J.L. - Susceptibility test for fungi: clinical and laboratorial correlations in medical mycology. Rev. Inst. Med. Trop. Sao Paulo, 57(Suppl 19): 57-64, 2015.

methodologies are simple, rapid, cost-effective and produce similar results to the reference methods for yeasts. Automated systems significantly reduce the biologist hands-on time, turnaround time, and variability due to the standardized format. Evaluation of these methodologies requires the determination of break point category agreements with reference methods. It is noteworthy that interpretative break points are only available for a few species of Candida, Cryptococcus and Aspergillus. Most of these tests have been able to detect in vitro resistance of Candida isolates, however some discrepancies have also been described. Reference procedures are irreplaceable nowadays to test and validate new antifungal agents, new methods and techniques, and the susceptibility profile of rare species which have not been evaluated by other methods ${ }^{27,29,42}$. Also, the increase of resistant strains associated with treatment failure highlights the need of antifungal resistance surveillance, which should ideally be made in reference laboratories using reference procedures.

\section{RESUMO}

\section{Teste de suscetibilidade para fungos: correlações clínico- laboratoriais em Micologia médica}

Nas últimas décadas, os testes de suscetibilidade a antifúngicos foram padronizados e, atualmente, servem tal como os testes de suscetibilidade a antibacterianos em laboratórios de microbiologia. Métodos de referência norte americanos e europeus foram desenvolvidos, assim como os equivalentes sistemas comerciais, estes últimos mais apropriados a laboratórios clínicos. A detecção de cepas resistentes por meio de tais sistemas permitiu o estudo e a compreensão das bases moleculares dos mecanismos de resistência de espécies fúngicas a fármacos antifúngicos. Além disso, foram realizados muitos estudos sobre a correlação de resultados obtidos in vitro com o desfecho clínico de pacientes permitindo a conclusão de que infecções por cepas resistentes têm pior evolução em relação às causadas por cepas sensíveis. Os estudos permitiram o estabelecimento de pontos de corte interpretativos (interpretative breakpoints development) para Candida spp. e Aspergillus spp., os agentes etiológicos mais frequentes de infecções fúngicas em todo o mundo. Em resumo, os testes de suscetibilidade representam uma ferramenta essencial para a orientação do tratamento de doenças fúngicas, para o conhecimento da epidemiologia local e global, bem como para a identificação de resistência a antifúngicos.

\section{REFERENCES}

1. Alastruey-Izquierdo A, Castelli MV, Cuesta I, Monzon A, Cuenca-Estrella M, RodriguezTudela JL. Activity of posaconazole and other antifungal agents against Mucorales strains identified by sequencing of internal transcribed spacers. Antimicrob Agents Chemother. 2009;53:1686-9.

2. Alastruey-Izquierdo A, Cuenca-Estrella M, Monzon A, Mellado E, Rodriguez-Tudela JL. Antifungal susceptibility profile of clinical Fusarium spp. isolates identified by molecular methods. J Antimicrob Chemother. 2008;61:805-9.

3. Alastruey-Izquierdo A, Cuenca-Estrella M, Monzón A, Rodriguez-Tudela JL. Prevalence and susceptibility testing of new species of Pseudallescheria and Scedosporium in a collection of clinical mold isolates. Antimicrob Agents Chemother. 2007;51:748-51.

4. Alcazar-Fuoli L, Mellado E. Current status of antifungal resistance and its impact on clinical practice. Br J Haematol. 2014;166:471-84
5. Alexander BD, Byrne TC, Smith KL, Hanson KE, Anstrom KJ, Perfect JR, et al. Comparative evaluation of Etest and sensititre YeastOne panels against the Clinical and Laboratory Standards Institute M27-A2 reference broth microdilution method for testing Candida susceptibility to seven antifungal agents. J Clin Microbiol. 2007;45:698-706.

6. Arendrup MC, Cuenca-Estrella M, Lass-Flörl C, Hope W, EUCAST-AFST. EUCAST technical note on the EUCAST definitive document EDef 7.2: method for the determination of broth dilution minimum inhibitory concentrations of antifungal agents for yeasts EDef 7.2 (EUCAST-AFST). Clin Microbiol Infect. 2012;18:E246-7.

7. Arendrup MC, Dzajic E, Jensen RH, Johansen HK, Kjaeldgaard P, Knudsen JD, et al. Epidemiological changes with potential implication for antifungal prescription recommendations for fungaemia: data from a nationwide fungaemia surveillance programme. Clin Microbiol Infect. 2013;19:E343-53.

8. Arendrup MC, Garcia-Effron G, Lass-Florl C, Lopez AG, Rodriguez-Tudela JL, CuencaEstrella M, et al. Echinocandin susceptibility testing of Candida species: comparison of EUCAST EDef 7.1, CLSI M27-A3, Etest, disk diffusion, and agar dilution methods with RPMI and isosensitest media. Antimicrob Agents Chemother. 2010;54:426-39.

9. Arendrup MC, Park S, Brown S, Pfaller M, Perlin DS. Evaluation of CLSI M44-A2 disk diffusion and associated breakpoint testing of caspofungin and micafungin using a well-characterized panel of wild-type and fks hot spot mutant Candida isolates. Antimicrob Agents Chemother. 2011;55:1891-5.

10. Arendrup MC, Pfaller MA, Danish Fungaemia Study Group. Caspofungin Etest susceptibility testing of Candida species: risk of misclassification of susceptible isolates of $C$. glabrata and $C$. krusei when adopting the revised CLSI caspofungin breakpoints. Antimicrob Agents Chemother. 2012;56:3965-8.

11. Barry AL, Pfaller MA, Rennie RP, Fuchs PC, Brown SD. Precision and accuracy of fluconazole susceptibility testing by broth microdilution, Etest, and disk diffusion methods. Antimicrob Agents Chemother. 2002;46:1781-4.

12. Brown GD, Denning DW, Gow NA, Levitz SM, Netea MG, White TC. Hidden killers: human fungal infections. Sci Transl Med. 2012;4:165rv13.

13. Canton E, Peman J, Iniguez C, Hervas D, Lopez-Hontangas JL, Pina-Vaz C, et al. Epidemiological cutoff values for fluconazole, itraconazole, posaconazole, and voriconazole for six Candida species as determined by the colorimetric Sensititre YeastOne method. J Clin Microbiol. 2013;51:2691-5.

14. Chryssanthou E, Cuenca-Estrella M. Comparison of the Antifungal Susceptibility Testing Subcommittee of the European Committee on Antibiotic Susceptibility Testing proposed standard and the E-test with the NCCLS broth microdilution method for voriconazole and caspofungin susceptibility testing of yeast species. J Clin Microbiol. 2002;40:3841-4.

15. Chryssanthou E, Cuenca-Estrella M. Comparison of the EUCAST-AFST broth dilution method with the CLSI reference broth dilution method (M38-A) for susceptibility testing of posaconazole and voriconazole against Aspergillus spp. Clin Microbiol Infect. 2006;12:901-4.

16. Clinical and Laboratory Standards Institute. Method for Antifungal Disk Diffusion Susceptibility Testing of Yeasts; Approved Guideline. CLSI document M44-A2. $2^{\text {nd }}$ ed. Wayne: Clinical and Laboratory Standards Institute; 2009.

17. Clinical and Laboratory Standards Institute. Method for Antifungal Disk Difusion Susceptibility Testing of Nondermatophyte Filamentous Fungi; Approved Guideline. CLSI document M51-A. Wayne: Clinical and Laboratory Standards Institute; 2010.

18. Clinical and Laboratory Standards Institute. Performance Standards for Antifungal Disk Diffusion Susceptibility Testing of Filamentous Fungi; Informational Supplement CLSI document M51-S1. Wayne: Clinical and Laboratory Standards Institute; 2010.

19. Clinical and Laboratory Standards Institute. Reference Method for Broth Dilution Antifungal Susceptibility Testing of Yeasts; Approved Standard. CLSI document M27-A. Wayne: Clinical and Laboratory Standards Institute; 1997. 
ALASTRUEY-IZQUIERDO, A.; MELHEM, M.S.C.; BONFIETTI, L.X. \& RODRIGUEZ-TUDELA, J.L. - Susceptibility test for fungi: clinical and laboratorial correlations in medical mycology. Rev. Inst. Med. Trop. Sao Paulo, 57(Suppl 19): 57-64, 2015.

20. Clinical and Laboratory Standards Institute. Reference Method for Broth Dilution Antifungal Susceptibility Testing of Yeast; Approved Standard-Third Edition. CLSI document M27-A3. Wayne: Clinical and Laboratory Standards Institute; 2008.

21. Clinical and Laboratory Standards Institute. Reference Method for Broth Dilution Antifungal Susceptibility Testing of Filamentous Fungi; Approved Standard-Second Edition. CLSI document M38-A2. Wayne: Clinical and Laboratory Standards Institute; 2008

22. Clinical and Laboratory Standards Institute. Reference method for broth dilution antifungal susceptibility testing of yeasts; $4^{\text {th }}$ Informational Supplement. CLSI document M27-S4. Wayne: Clinical and Laboratory Standards Institute; 2012

23. Clinical and Laboratory Standards Institute. Zone Diameter Interpretive Standards, Corresponding Minimal Inhibitory Concentration (MIC) Interpretive Breakpoints, and Quality Control Limits for Antifungal Disk Diffusion Susceptibility Testing of Yeasts; Third Informational Supplement. CLSI document M44-S3. Wayne: Clinical and Laboratory Standards Institute; 2009

24. Colombo AL, Tobón A, Restrepo A, Queiroz-Telles F, Nucci M. Epidemiology of endemic systemic fungal infections in Latin America. Med Mycol. 2011;49:785-98.

25. Cornely OA, Bassetti M, Calandra T, Garbino J, Kullberg BJ, Lortholary O, et al. ESCMID* guideline for the diagnosis and management of Candida diseases 2012: non-neutropenic adult patients. Clin Microbiol Infect. 2012;18(Suppl 7):19-37.

26. Cuenca-Estrella M, Alastruey-Izquierdo A, Alcazar-Fuoli L, Bernal-Martinez L, GomezLopez A, Buitrago MJ, et al. In vitro activities of 35 double combinations of antifungal agents against Scedosporium apiospermum and Scedosporium prolificans. Antimicrob Agents Chemother. 2008;52:1136-9.

27. Cuenca-Estrella M, Gomez-Lopez A, Alastruey-Izquierdo A, Bernal-Martinez L, Cuesta I, Buitrago MJ, et al. Comparison of the Vitek 2 antifungal susceptibility system with the Clinical and Laboratory Standards Institute (CLSI) and European Committee on Antimicrobial Susceptibility Testing (EUCAST) Broth Microdilution Reference Methods and with the Sensititre Yeast One and Etest techniques for in vitro detection of antifungal resistance in yeast isolates. J Clin Microbiol. 2010;48:1782-6.

28. Cuenca-Estrella M, Gomez-Lopez A, Mellado E, Rodriguez-Tudela JL. Correlation between the procedure for antifungal susceptibility testing for Candida spp. of the European Committee on Antibiotic Susceptibility Testing (EUCAST) and four commercial techniques. Clin Microbiol Infect. 2005;11:486-92.

29. Cuenca-Estrella M, Rodriguez-Tudela JL. The current role of the reference procedures by CLSI and EUCAST in the detection of resistance to antifungal agents in vitro. Expert Rev Anti Infect Ther. 2010;8:267-76.

30. Cuenca-Estrella M, Ruiz-Diez B, Martinéz-Suaréz JV, Monzón A, Rodriguez-Tudela JL. Comparative in-vitro activity of voriconazole (UK-109,496) and six other antifungal agents against clinical isolates of Scedosporium prolificans and Scedosporium apiospermum. J Antimicrob Chemother.1999;43:149-51.

31. Dannaoui E, Desnos-Ollivier M, Garcia-Hermoso D, Grenouillet F, Cassaing S, Baixench MT, et al. Candida spp. with acquired echinocandin resistance, France, 2004-2010. Emerg Infect Dis. 2012;18:86-90.

32. Dias AL, Matsumoto FE, Melhem MS, da Silva EG, Auler ME, de Siqueira AM, et al. Comparative analysis of Etest and broth microdilution method (AFST-EUCAST) for trends in antifungal drug susceptibility testing of Brazilian Cryptococcus neoformans isolates. J Med Microbiol. 2006;55(Pt 12):1693-9.

33. Ellis D. Amphotericin B: spectrum and resistance. J Antimicrob Chemother. 2002;49(Suppl 1):7-10

34. Espinel-Ingroff A, Arthington-Skaggs B, Iqbal N, Ellis D, Pfaller MA, Messer S, et al Multicenter evaluation of a new disk agar diffusion method for susceptibility testing of filamentous fungi with voriconazole, posaconazole, itraconazole, amphotericin B, and caspofungin. J Clin Microbiol. 2007;45:1811-20.
35. Espinel-Ingroff A, Canton E, Gibbs D, Wang A. Correlation of Neo-Sensitabs tablet diffusion assay results on three different agar media with CLSI broth microdilution M27-A2 and disk diffusion M44-A results for testing susceptibilities of Candida spp. and Cryptococcus neoformans to amphotericin B, caspofungin, fluconazole, itraconazole, and voriconazole. J Clin Microbiol. 2007;45:858-64.

36. Espinel-Ingroff A. Comparison of three commercial assays and a modified disk diffusion assay with two broth microdilution reference assays for testing zygomycetes, Aspergillus spp., Candida spp., and Cryptococcus neoformans with posaconazole and amphotericin B. J Clin Microbiol. 2006;44:3616-22.

37. Forastiero A, Mesa-Arango AC, Alastruey-Izquierdo A, Alcazar-Fuoli L, Bernal-Martinez $\mathrm{L}$, Pelaez T, et al. Candida tropicalis antifungal cross-resistance is related to different azole target (Erg11p) modifications. Antimicrob Agents Chemother. 2013;57:4769-81.

38. Gupta P, Khare V, Kumar D, Ahmad A, Banerjee G, Singh M. Comparative Evaluation of Disc Diffusion and E-test with Broth Micro-dilution in Susceptibility testing of Amphotericin B, Voriconazole and Caspofungin against Clinical Aspergillus isolates. J Clin Diagn Res. 2015;9:DC04-7.

39. Havlickova B, Czaika VA, Friedrich M. Epidemiological trends in skin mycoses worldwide. Mycoses. 2008;51(Suppl 4):2-15.

40. Koehling HL, Willinger B, Buer J, Rath PM, Steinmann J. Comparative evaluation of a new commercial colorimetric microdilution assay (SensiQuattro Candida EU) with MIC test strip and EUCAST broth microdilution methods for susceptibility testing of invasive Candida isolates. J Clin Microbiol. 2014;53:255-61.

41. Lass-Flörl C. The changing face of epidemiology of invasive fungal disease in Europe. Mycoses. 2009;52:197-205.

42. Marcos-Zambrano LJ, Escribano P, Rueda C, Zaragoza Ó, Bouza E, Guinea J. Comparison between the EUCAST procedure and the Etest for determination of the susceptibility of Candida species isolates to micafungin. Antimicrob Agents Chemother. 2013;57:5767-70.

43. Melhem MS, Bertoletti A, Lucca HR, Silva RB, Meneghin FA, Szeszs MW. Use of the VITEK 2 system to identify and test the antifungal susceptibility of clinically relevant yeast species. Braz J Microbiol. 2014;44:1257-66.

44. Mesa-Arango AC, Scorzoni L, Zaragoza O. It only takes one to do many jobs Amphotericin B as antifungal and immunomodulatory drug. Front Microbiol. 2012;3:286.

45. Park BJ, Wannemuehler KA, Marston BJ, Govender N, Pappas PG, Chiller TM. Estimation of the current global burden of cryptococcal meningitis among persons living with HIV/AIDS. AIDS. 2009;23:525-30

46. Patel GP, Simon D, Scheetz M, Crank CW, Lodise T, Patel N. The effect of time to antifungal therapy on mortality in Candidemia associated septic shock. Am J Ther. 2009;16:508-11

47. Peterson JF, Pfaller MA, Diekema DJ, Rinaldi MG, Riebe KM, Ledeboer NA Multicenter comparison of the Vitek 2 antifungal susceptibility test with the CLSI broth microdilution reference method for testing caspofungin, micafungin, and posaconazole against Candida spp. J Clin Microbiol. 2011;49:1765-71.

48. Pfaller MA, Chaturvedi V, Diekema DJ, Ghannoum MA, Holliday NM, Killian SB, et al. Comparison of the Sensititre YeastOne colorimetric antifungal panel with CLSI microdilution for antifungal susceptibility testing of the echinocandins agains Candida spp., using new clinical breakpoints and epidemiological cutoff values. Diagn Microbiol Infect Dis. 2012;73:365-8.

49. Pfaller MA, Diekema DJ, Andes D, Arendrup MC, Brown SD, Lockhart SR, et al. Clinical breakpoints for the echinocandins and Candida revisited: integration of molecular clinical, and microbiological data to arrive at species-specific interpretive criteria. Drug Resist Updat. 2011;14:164-76. 
ALASTRUEY-IZQUIERDO, A.; MELHEM, M.S.C.; BONFIETTI, L.X. \& RODRIGUEZ-TUDELA, J.L. - Susceptibility test for fungi: clinical and laboratorial correlations in medical mycology. Rev. Inst. Med. Trop. Sao Paulo, 57(Suppl 19): 57-64, 2015.

50. Pfaller MA, Diekema DJ, Ostrosky-Zeichner L, Rex JH, Alexander BD, Andes D, et al. Correlation of MIC with outcome for Candida species tested against caspofungin, anidulafungin, and micafungin: analysis and proposal for interpretive MIC breakpoints. J Clin Microbiol. 2008;46:2620-9.

51. Pfaller MA, Diekema DJ, Procop GW, Wiederhold NP. Multicenter evaluation of the new Vitek 2 yeast susceptibility test using new CLSI clinical breakpoints for fluconazole. J Clin Microbiol. 2014;52:2126-30.

52. Pfaller MA, Diekema DJ, Rex JH, Espinel-Ingroff A, Johnson EM, Andes D, et al. Correlation of MIC with outcome for Candida species tested against voriconazole: analysis and proposal for interpretive breakpoints. J Clin Microbiol. 2006;44:819-26.

53. Pfaller MA. Antifungal drug resistance: mechanisms, epidemiology, and consequences for treatment. Am J Med. 2012;125(1 Suppl):S3-13 .

54. Rodriguez-Tudela JL, Berenguer J, Guarro J, Kantarcioglu AS, Horre R, de Hoog GS, et al. Epidemiology and outcome of Scedosporium prolificans infection, a review of 162 cases. Med Mycol. 2009;47:359-70.

55. Rodriguez-Tudela JL, Donnelly JP, Pfaller MA, Chryssantou E, Warn P, Denning DW, et al. Statistical analyses of correlation between fluconazole MICs for Candida spp. assessed by standard methods set forth by the European Committee on Antimicrobial Susceptibility Testing (E.Dis. 7.1) and CLSI (M27-A2). J Clin Microbiol. 2007;45:109-11.
56. Snelders E, Huis In 't Veld RA, Rijs AJ, Kema GH, Melchers WJ, Verweij PE. Possible environmental origin of resistance of Aspergillus fumigatus to medical triazoles. Appl Environ Microbiol. 2009;75:4053-7.

57. Subcommittee on Antifungal Susceptibility Testing of the ESCMID European Committee for Antimicrobial Susceptibility Testing. EUCAST Technical Note on the method for the determination of broth dilution minimum inhibitory concentrations of antifungal agents for conidia-forming moulds. Clin Microbiol Infect. 2008;14:982-4.

58. Ullmann AJ, Akova M, Herbrecht R, Viscoli C, Arendrup MC, Arikan-Akdagli S, $e$ al. ESCMID* guideline for the diagnosis and management of Candida diseases 2012: adults with haematological malignancies and after haematopoietic stem cell transplantation (HCT). Clin Microbiol Infect. 2012;18(Suppl 7):53-67.

59. Zhanel GG, Karlowsky JA, Harding GA, Balko TV, Zelenitsky SA, Friesen M, et al. In vitro activity of a new semisynthetic echinocandin, LY-303366, against systemic isolates of Candida species, Cryptococcus neoformans, Blastomyces dermatitidis, and Aspergillus species. Antimicrob Agents Chemother. 1997;41:863-5. 